\title{
Physicochemical Properties of Acylglutamic Acid- Alkylamine Complexes in Aqueous Media
}

\author{
Ryo Yoshikawa ${ }^{1}$, Masaaki Akamatsu ${ }^{1}$, Kenichi Sakai $^{1,2 *}$, and Hideki Sakai ${ }^{1,2}$ \\ ${ }^{1}$ Department of Pure and Applied Chemistry, Faculty of Science and Technology, Tokyo University of Science, 2641 Yamazaki, Noda, Chiba \\ 278-8510, JAPAN \\ ${ }^{2}$ Research Institute for Science and Technology, Tokyo University of Science, 2641 Yamazaki, Noda, Chiba 278-8510, JAPAN
}

\begin{abstract}
We studied the physicochemical properties of 1:1 stoichiometric complexes of acylglutamic acids (CnGlu) with tertiary alkylamines (CnDMA) in water at their low and high concentrations. Static surface tensiometry suggested that the critical micelle concentration $(\mathrm{cmc})$ decreased with increasing hydrophobic chain length of the complexes. In addition, CnGlu-CnDMA yielded lower cme than the C12Glu single system. In the region of high concentrations, several phase states including isotropic liquid $\left(L_{1}\right)$ phase, hexagonal liquid crystal $\left(\mathrm{H}_{1}\right)$ phase, bicontinuous cubic liquid crystal $\left(\mathrm{V}_{1}\right)$ phase, and lamellar liquid crystal $\left(L_{a}\right)$ phase were observed. Assemblies with lesser positive curvature tend to be formed with increasing complex concentration, increasing temperature, and increasing hydrophobic chain length. Additionally, the complex formation resulted in the molecular assemblies with lesser positive curvature.
\end{abstract}

Key words: acylglutamic acid, alkylamine, gemini surfactant, surface tension, liquid crystal

\section{Introduction}

Gemini surfactant is a dimer of monomeric surfactants linked by a space unit, being composed of two hydrophilic headgroups and two hydrophobic chains in a molecule ${ }^{1,2}$. Gemini surfactants have been attracted as a new type of surfactants because of their excellent micellization efficiency at low concentrations and good solubility in water ${ }^{1,}$ ${ }^{2)}$. Thus, gemini surfactants are generally categorized into an eco-friendly surfactant. However, their synthesis and purification processes are usually complicated, and this leads to their high selling price in the market.

Based on these backgrounds, functionally-equivalent materials with gemini surfactants have been investigated, including dicarboxylic acid-alkylamine (or diamine-alkyl carboxylic acid) complexes, as shown in the following references. The stoichiometric complex formation is simply based on the proton transfer from the acid to the amine. This does not lead to an increase in the ionic strength in solution, which is significantly different from systems of catanionic (cationic and anionic) surfactant mixtures. They are sometimes called "gemini-like amphiphile ${ }^{3)}$, " pseudogemini surfactant $t^{4-6)}$ ”, or "counterion-coupled gemini surfactant $(\operatorname{cocogem})^{7,8)}$ ". Such noncovalent-type amphiphiles have been investigated in the aspect of surface tension in water $^{7)}$ and rheology based on the formation of wormlike micelles $^{9)}$ or hydrogels ${ }^{10)}$. Recently, reversible control of stability of emulsion ${ }^{11)}$ was also achieved in the system of citric acid-alkylamidodimethyl amine complex. However, we find a lack of knowledge about their phase behaviors at low and high concentrations in water, which is an important and novel subject for reporting.

In our previous work, we reported that acylglutamic acid-alkylamine complexes (CnGlu-C $n$ DMA, where $n$ is the number of carbon atoms in the hydrophobic chains) form $\mathrm{pH}$-sensitive wormlike micelles and hydrogels ${ }^{12)}$. In addition, these complexes can stabilize oil droplets dispersed in water against their coalescence ${ }^{13)}$. In this paper, we report the physicochemical property of $\mathrm{C} n \mathrm{Glu}-\mathrm{C} n \mathrm{DMA}$ in their dilute aqueous solutions by static surface tensiometry. We also demonstrate the phase behavior of $\mathrm{C} n \mathrm{Glu}-\mathrm{C} n \mathrm{DMA}$ in water on the basis of polarized optical microscopy (POM) and small angle X-ray scattering (SAXS) results.

\section{Experimental Section}

\subsection{Materials.}

Dodecanoylglutamic acid (C12Glu) and CnGlu-CnDMA, ( $n=10,12$, and 14$)$ were synthesized according to the same procedure mentioned in our previous paper ${ }^{14}$. Figure

\footnotetext{
*Correspondence to: Kenichi Sakai, Department of Pure and Applied Chemistry, Faculty of Science and Technology, Tokyo University of Science, 2641 Yamazaki, Noda, Chiba 278-8510, JAPAN

E-mail: k-sakai@rs.tus.ac.jp

Accepted March 31, 2020 (received for review February 4, 2020)

Journal of Oleo Science ISSN 1345-8957 print / ISSN 1347-3352 online

http://www.jstage.jst.go.jp/browse/jos/ http://mc.manusriptcentral.com/jjocs
} 


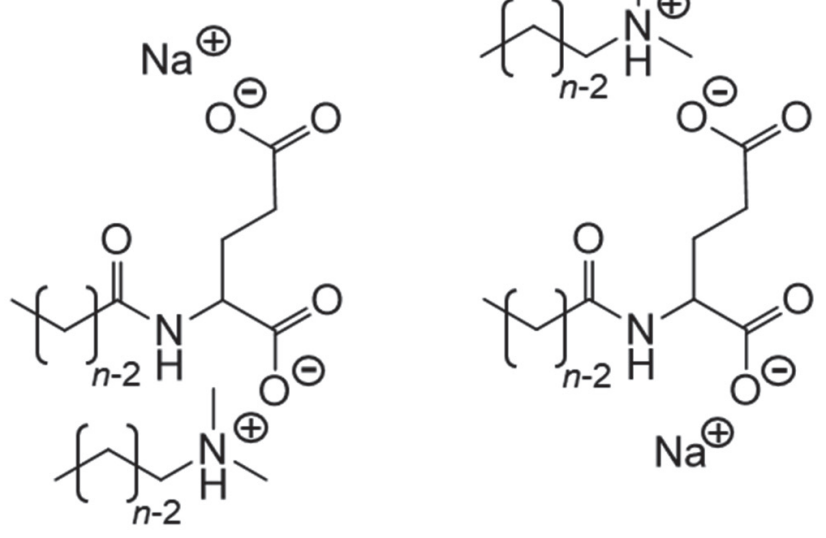

Fig. 1 Chemical structures of CnGlu-CnDMA $(n=10,12$, and 14) neutralized by $\mathrm{NaOH}$. CnGlu-CnDMA was always neutralized by $\mathrm{NaOH}$ at a fixed mole ratio, CnGlu-CnDMA:NaOH = 1:1.

1 shows chemical structures of CnGlu-CnDMA neutralized by $\mathrm{NaOH}$. $1 \mathrm{~mol} / \mathrm{L} \mathrm{NaOH}$ aqueous solution was purchased from Fujifilm Wako Pure Chemical Co. The water used in this study was deionized with a Barnstead NANO Pure DIamond UV system and filtered with a Millipore membrane filter $(0.22 \mu \mathrm{m}$ pore size $)$.

\subsection{Preparations and instrumentations}

In our previous papers ${ }^{12,14)}$, we reported the solubility of CnGlu-CnDMA in their aqueous solutions as a function of pH. In our present study, CnGlu-CnDMA was always neutralized by $\mathrm{NaOH}$ at a fixed mole ratio, CnGlu-CnDMA: $\mathrm{NaOH}=1: 1$.

The Krafft point of C12Glu monosodium salt is reported as $38.1^{\circ} \mathrm{C}^{15)}$, being higher than room temperature $\left(25^{\circ} \mathrm{C}\right)$. This means that the surfactant precipitates in water at $25^{\circ} \mathrm{C}$. Therefore, C12Glu was neutralized by $\mathrm{NaOH}$ at a mole ratio, $\mathrm{C} 12 \mathrm{Glu}: \mathrm{NaOH}=1: 2$.

Static surface tension measurements were performed using a Krüss K100C Wilhelmy auto surface tensiometer with a platinum plate. The surface tension was assumed to be equilibrated when the change in the surface tension became less than $0.01 \mathrm{mN} \mathrm{m}^{-1}$ per $90 \mathrm{~s}$. The measurement temperature was set at $25^{\circ} \mathrm{C}$.

Mixtures composed of CnGlu-CnDMA and water were prepared as follows. The mixtures were sealed in a glass test tube. The system was heated up to $80^{\circ} \mathrm{C}$ using a temperature-controlled water bath for $30 \mathrm{~min}$, mixed at a constant rotation speed of 3,000 rpm for 3 min using a vortex mixer, centrifuged for $30 \mathrm{~min}$ at a constant rotation speed of 3,500 rpm using a Kokusan H11-NB centrifuge, and finally the equilibration cycle was repeated 3 times. Then, the system was left for 2 weeks in an incubator set at a constant temperature of $25^{\circ} \mathrm{C}$. The phase state was examined after this equilibration period.

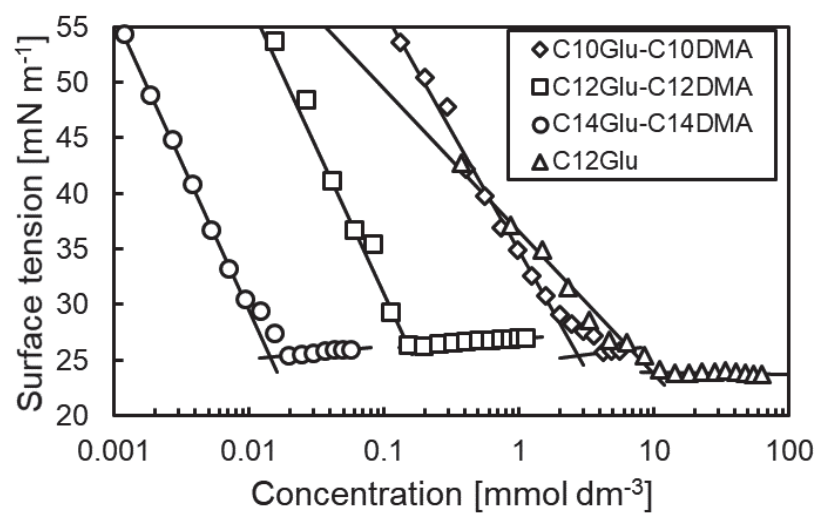

Fig. 2 Surface tension - concentration plots for the CnGlu-CnDMA and C12Glu systems.

POM observations were performed at $25^{\circ} \mathrm{C}$ using an Olympus IX73 microscope.

SAXS measurements were carried out using an Anton Paar SAXSess camera equipped with a PANalytical PW3830 laboratory X-ray generator, a multilayer film Goebel mirror, a block collimator, a semi-transmissible beam stop, a TCS120 temperature controller, and an imaging plate detector. The apparatus was operated at $40 \mathrm{kV}$ and $50 \mathrm{~mA}$ using $\mathrm{Cu}$-K $\alpha$ X-rays (wavelength of $0.154 \mathrm{~nm}$ ). The measurement time was fixed at $10 \mathrm{~min}$. The measurement temperatures were in the range $25-65^{\circ} \mathrm{C}$.

\section{Results and Discussion}

\subsection{Aqueous solution properties at low concentrations}

The CnGlu-CnDMA complex is a mixture of the two possible isomers shown in Fig. 1. Furthermore, the complex is a dynamic amphiphile. This means that the complex formation/deformation(in other words, the complex-monomer transition) would continually occur not only in the solution phase but also at the air/solution interface. Figure 2 shows the surface tension results for the aqueous CnGlu-CnDMA and C12Glu solutions as a function of their concentrations. For all of the analogs, the surface tension decreased with increasing concentration, and attained a plateau level at a given concentration (critical micelle concentration, cmc). Table 1 shows the interfacial parameters calculated from the results; cmc, surface tension measured at the cmc $\left(\gamma_{\mathrm{cmc}}\right)$, surface excess concentration estimated at the cmc $\left(\Gamma_{\text {cmc }}\right)$, and occupied area per CnGlu-CnDMA (or C12Glu) adsorbed at the air/aqueous solution interface $\left(A_{\mathrm{cmc}}\right) . \Gamma_{\mathrm{cmc}}$ and $A_{\text {cmc }}$ values were calculated according to the following equations;

$$
\Gamma_{\mathrm{cmc}}=-\frac{1}{2.30 i R T}\left(\frac{d \gamma}{d \log C}\right)_{T}
$$


Table 1 Interfacial parameters calculated from the surface tension data.

\begin{tabular}{ccccc}
\hline System & $\begin{array}{c}\mathrm{cmc} \\
{\left[\mathrm{mmol} \mathrm{dm}^{-3}\right]}\end{array}$ & $\begin{array}{c}\gamma_{\mathrm{cmc}} \\
{\left[\mathrm{mN} \mathrm{m}^{-1}\right]}\end{array}$ & $\begin{array}{c}\Gamma_{\mathrm{cmc}} \\
{\left[\mu \mathrm{mol} \mathrm{m}^{-2}\right]}\end{array}$ & $\begin{array}{c}A_{\mathrm{cmc}} \\
{\left[\mathrm{nm}^{2}\right]}\end{array}$ \\
\hline C10Glu-C10DMA & 2.7 & 25 & 1.3 & 1.3 \\
C12Glu-C12DMA & 0.15 & 26 & 1.5 & 1.1 \\
C14Glu-C14DMA & 0.014 & 25 & 1.6 & 1.1 \\
C12Glu & 9.7 & 24 & 0.75 & 2.2 \\
\hline
\end{tabular}

$$
A_{\mathrm{cmc}}=\frac{1}{N_{A} \Gamma_{\mathrm{cmc}}}
$$

where, $R$ is the gas constant, $T$ is the absolute temperature, and $N_{A}$ is the Avogadro number. Additionally, $i$ is the number of adsorption species. In this study, $\Gamma_{\mathrm{cmc}}$ and $A_{\mathrm{cmc}}$ values were calculated under the assumption $i=3$, although the dissociation constant of $\mathrm{C} n \mathrm{Glu}-\mathrm{C} n \mathrm{DMA}$ in the solution phase has not been known, yet.

The cmc of $\mathrm{C} n \mathrm{Glu}-\mathrm{C} n \mathrm{DMA}$ decreased with increasing chain length, as expected. The increased chain length resulted in the increased hydrophobicity of CnGlu-CnDMA, leading to the decrease in cmc. This behavior is generally observed in many surfactant systems ${ }^{16)}$, including the $\mathrm{C} n$ Glu analogs ${ }^{15}$ ) and the carboxylic acid-alkylamine complex systems ${ }^{5,7)}$. In general, the linear relationship between $n$ and cmc is present for surfactant systems ${ }^{17)}$.

$$
\log \mathrm{cmc}=A-B n
$$

where $A$ and $B$ are constant. Figure 3 show the log cmc - $n$ plot for the CnGlu-C $n$ DMA and $\mathrm{C} n$ Glu disodium salts ${ }^{15}$ systems. Here, we plotted the log cmc as a function of $n$ (CnGlu) or $2 n$ (CnGlu-CnDMA), based on the expectation that the chain length of CnDMA also affects the cmc, even if $\mathrm{C} n$ DMA merely acts as a counterion and dissociates from $\mathrm{C} n \mathrm{Glu}$. The cmc decreased linearly with increasing $n$ or $2 n$, as expected. The slope was greater for CnGlu-CnDMA than for $\mathrm{C} n \mathrm{Glu}$, suggesting the larger impact of hydrophobic chain length on the cmc for the CnGlu-CnDMA analogs.

As shown in Table 1, the $A_{\text {cmc }}$ values estimated for $\mathrm{C} n \mathrm{G}$ lu-C $n$ DMA were much smaller than that for C12Glu. CnDMA acts as not only a counterion of CnGlu but also a hydrophobicity enhancer of the complex. As a result of these effects, the packing density of the complexes became larger at the air/solution interface than that of C12Glu.

\subsection{Phase behavior at high concentrations}

As stated in the Experimental section, the aqueous phase behavior was studied through POM and SAXS measurements. The temperature-concentration phase diagrams of $\mathrm{C} n$ Glu-CnDMA are summarized in Fig. 4. We discussed their phase behaviors as a function of(i) concentration, (ii) temperature, (iii)hydrophobic chain length, and (iv) complex formation with comparison for the reported C12Glu system ${ }^{18)}$.

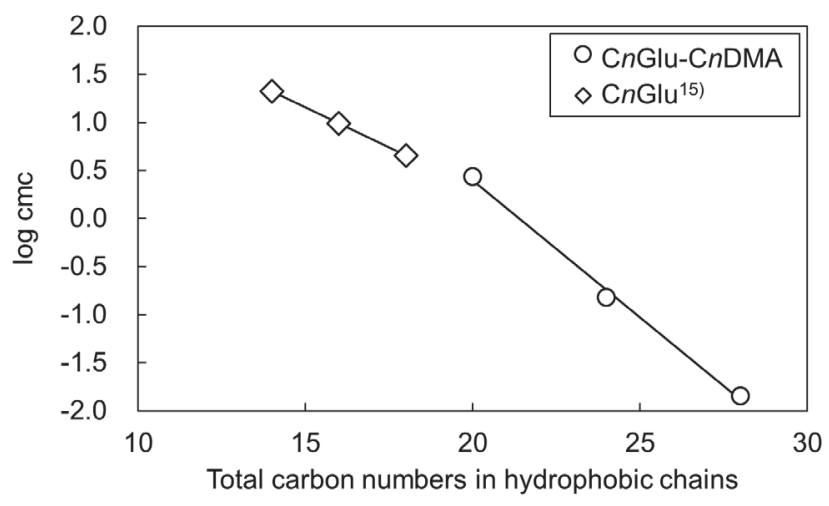

Fig. 3 Relationship between cmc and $n$ or $2 n$ (total carbon numbers in hydrophobic chains).

(i) Figure 5 shows typical SAXS and POM results obtained for the (a) C10Glu-C10DMA, (b) C12Glu-C12DMA, and (c) C14Glu-C14DMA systems at various concentrations at a constant temperature of $25^{\circ} \mathrm{C}$. In the case of C10GluC10DMA (Fig. 5a), the phase states were determined as follows: hexagonal liquid crystal $\left(\mathrm{H}_{1}\right)$ phase at $36 \mathrm{wt} \%$ (indicated by the ratio of $q$ values $=1: \sqrt{3}: 2$, the fan-like texture observed in the POM image, and its optically anisotropic nature), $\mathrm{H}_{1}$ and bicontinuous cubic liquid crystal $\left(\mathrm{V}_{1}\right)$ phases at 55 wt $\%$ (indicated by the ratio of $q$ values $=\sqrt{6}$ : $\sqrt{8}$, the fan-like texture observed in the POM image, and its optically anisotropic nature), $\mathrm{V}_{1}$ phase at $70 \mathrm{wt} \%$ (indicated by the ratio of $q$ values $=\sqrt{6}: \sqrt{8}$, and its optically isotropic nature), and lamellar liquid $\operatorname{crystal}\left(\mathrm{L}_{\alpha}\right)$ phase at 73 wt \% (indicated by the ratio of $q$ values $=1: 2$, the Maltesecross texture observed in the POM image, and its optically anisotropic nature).

Similarly, the $\mathrm{H}_{1}$ phase was identified in the cases of C12Glu-C12DMA (Fig. 5b) and C14Glu-C14DMA (Fig. 5c). However, the SAXS pattern yielded splitting of the peaks above the concentration of 54 wt\% (C12Glu-C12DMA) and 48 wt\% (C14Glu-C14DMA). This means the coexistence of two lyotropic liquid crystals. These complexes have a gelliquid crystal phase transition temperature, and the temperature increases with hydrophobic chain length ${ }^{13)}$. This causes a difficulty in preparing a homogenous sample at high concentrations and at low temperatures, under a given homogenization process.

In summary, the increased concentration resulted in the 

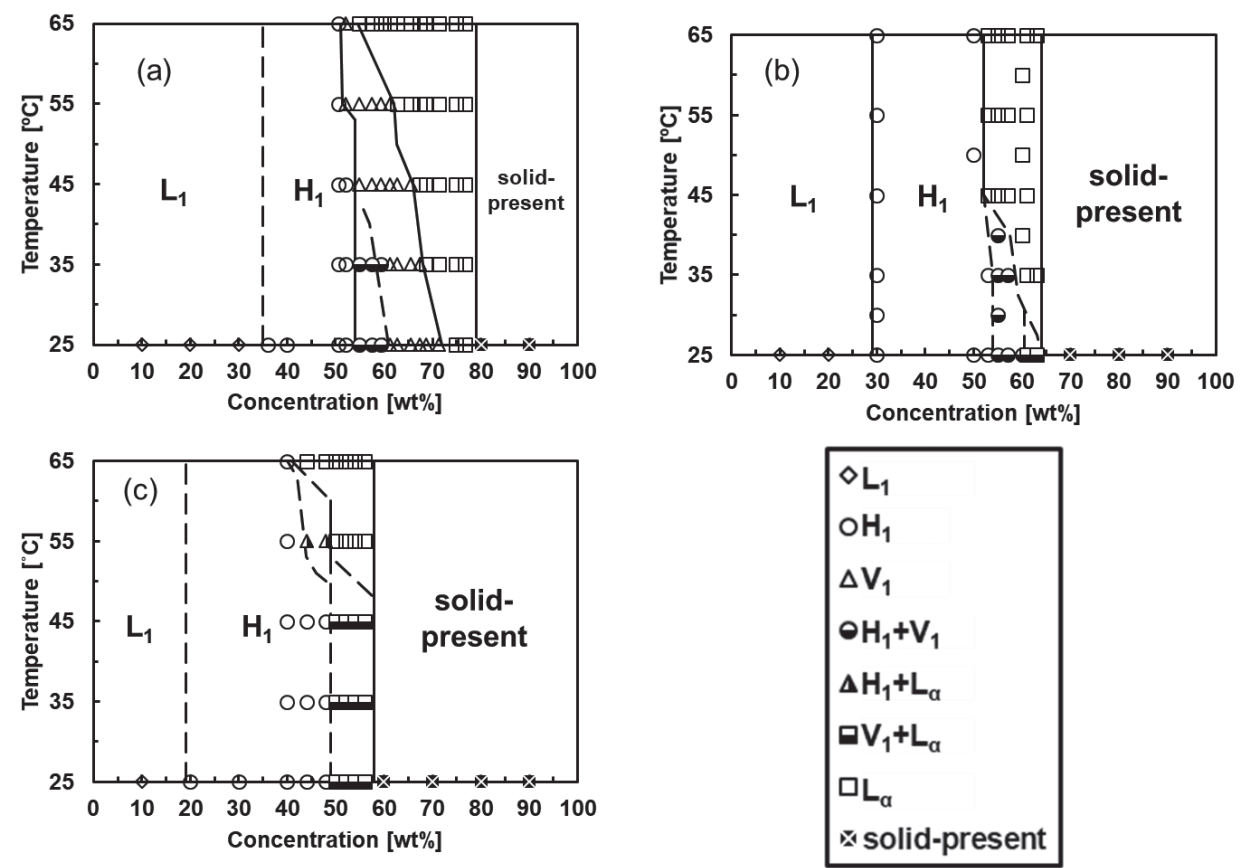

Fig. 4 Temperature-concentration phase diagrams of(a)C10Glu-C10DMA, (b)C12Glu-C12DMA, and (c) C14Glu-C14DMA. The complex samples were neutralized by 1 eq. $\mathrm{NaOH}$.
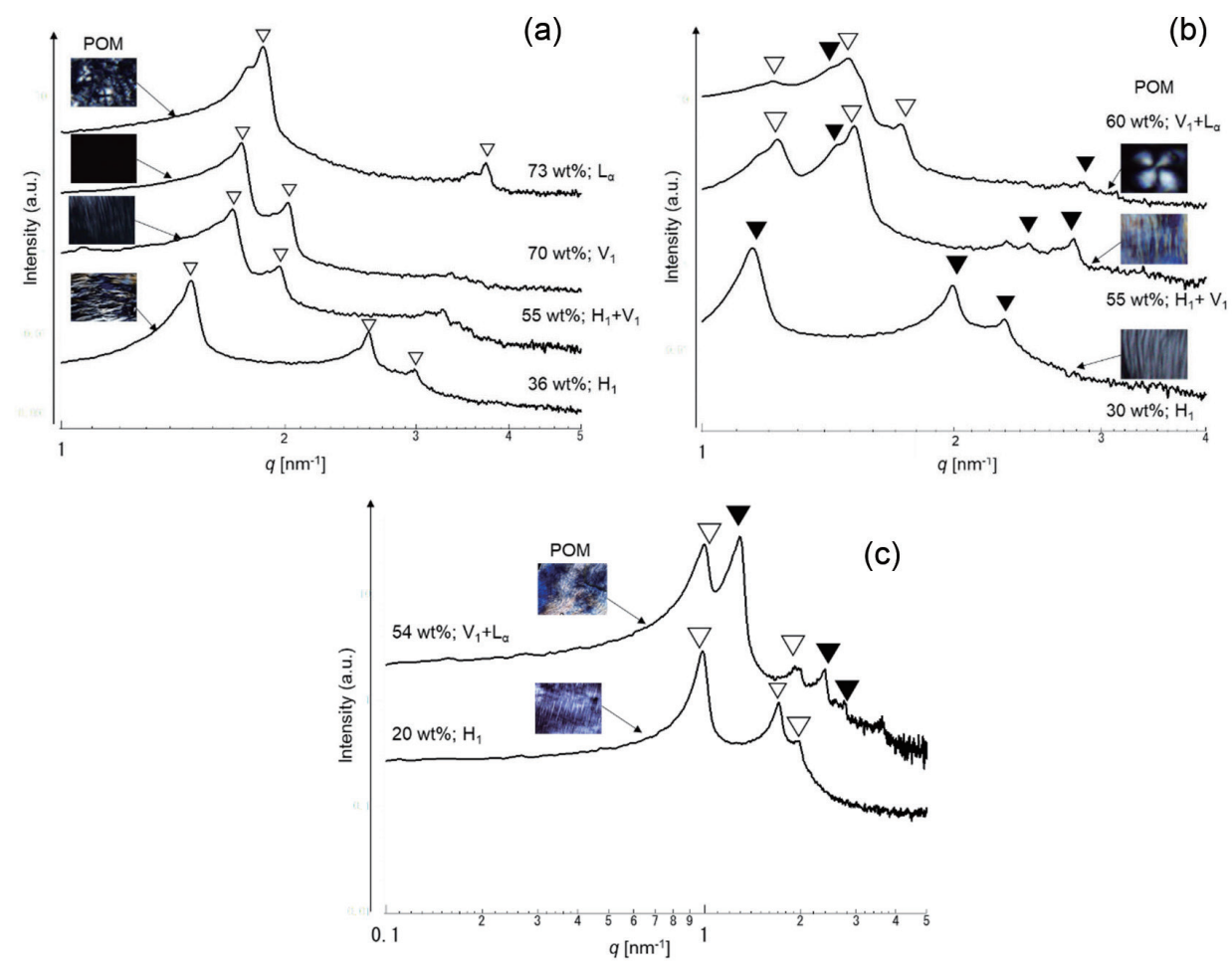

Fig. 5 POM images and SAXS patterns obtained for the (a)C10Glu-C10DMA, (b) C12Glu-C12DMA, and (c) C14Glu-C14DMA systems at various concentrations at a constant temperature of $25^{\circ} \mathrm{C}$. The characteristic ratios of $q$ values and POM images are as follows: (b) $\mathrm{H}_{1}$ phase at $30 \mathrm{wt} \%\left(1: \sqrt{3}: 2\right.$, the fan-like texture), $\mathrm{H}_{1}+\mathrm{V}_{1}$ phases at $55 \mathrm{wt} \%(1: \sqrt{3}: 2$ and $\sqrt{2}: \sqrt{3}$, the fan-like texture), and $\mathrm{V}_{1}+\mathrm{L}_{\alpha}$ phases at $60 \mathrm{wt} \%(\sqrt{2}: \sqrt{3}: \sqrt{4}$ and $1: 2$, the Maltese-cross texture); and $(\mathrm{c}) \mathrm{H}_{1}$ phase at $20 \mathrm{wt} \%(1: \sqrt{3}: 2$, the fan-like texture $)$, and $\mathrm{V}_{1}+\mathrm{L}_{\alpha}$ phases at $54 \mathrm{wt} \%(\sqrt{6}: \sqrt{20}: \sqrt{26}$ and $1: 2)$. The characterization information of (a) is shown in the text. 


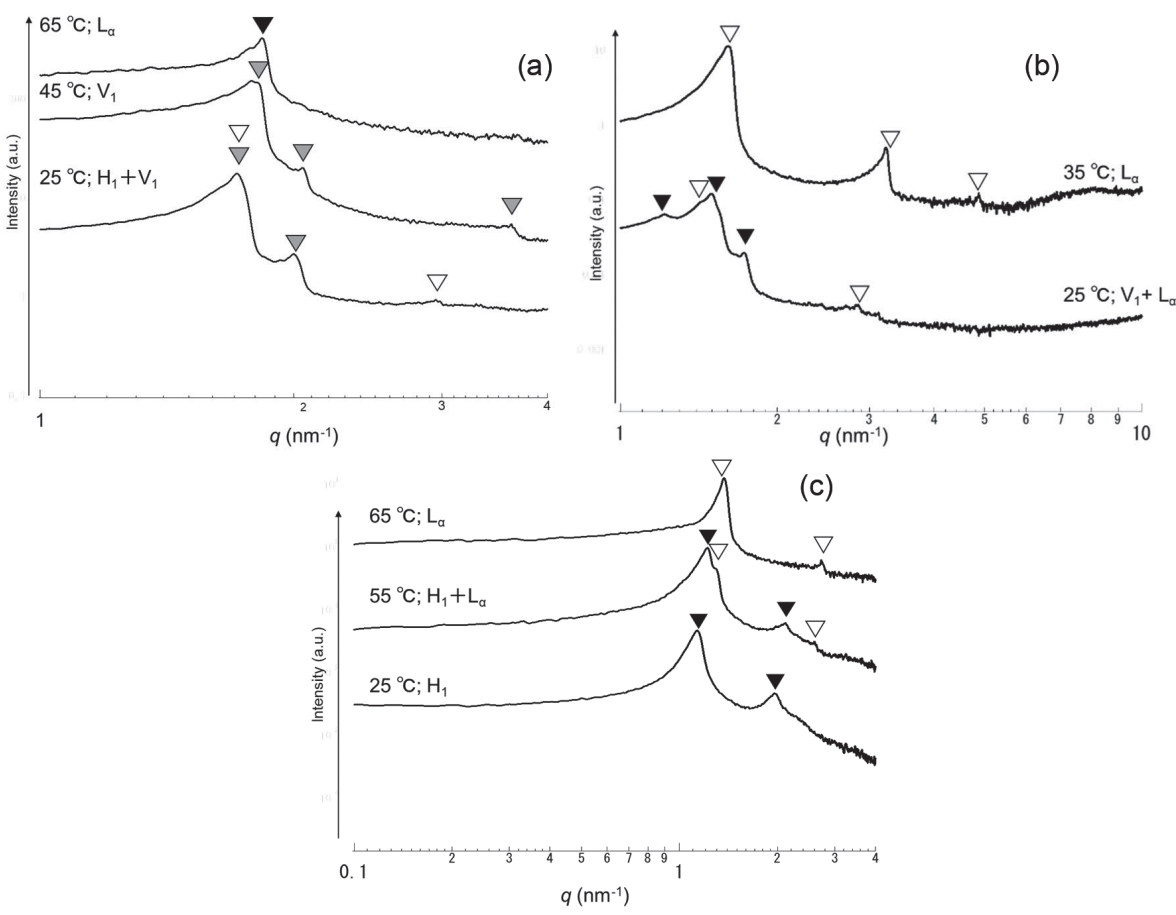

Fig. 6 SAXS patterns obtained for (a) C10Glu-C10DMA at a fixed concentration of 55 wt \%, (b) C12Glu-C12DMA at a fixed concentration of $60 \mathrm{wt} \%$, and (c) C14Glu-C14DMA at a fixed concentration of $44 \mathrm{wt} \%$ at various temperatures. The characteristic ratios of $q$ values are as follows: (a) $\mathrm{H}_{1}+\mathrm{V}_{1}$ phases at $25^{\circ} \mathrm{C}(1: \sqrt{3}$ and $\sqrt{6}: \sqrt{8}), \mathrm{V}_{1}$ phase at $45^{\circ} \mathrm{C}(\sqrt{6}$ : $\sqrt{8}: \sqrt{24})$, and $\mathrm{L}_{\alpha}$ phase at $65^{\circ} \mathrm{C}$; (b) $\mathrm{V}_{1}+\mathrm{L}_{\alpha}$ phases at $25^{\circ} \mathrm{C}(\sqrt{2}: \sqrt{3}: \sqrt{4}$ and $1: 2)$, and $\mathrm{L}_{\alpha}$ phase at $35^{\circ} \mathrm{C}(1: 2: 3)$; and $(\mathrm{c}) \mathrm{H}_{1}$ phase at $25^{\circ} \mathrm{C}(1: \sqrt{3}), \mathrm{H}_{1}+\mathrm{L}_{\alpha}$ phases at $55^{\circ} \mathrm{C}(1: \sqrt{3}$ and $1: 2)$, and $\mathrm{L}_{\alpha}$ phase at $65^{\circ} \mathrm{C}(1: 2)$.

phase transitions as $\mathrm{L}_{1}-\mathrm{H}_{1}-\mathrm{V}_{1}-\mathrm{L}_{\alpha}$ for all of the complexes. This means that the increased complex concentration yields molecular assemblies with lesser positive curvature, resulting from a decreased cross-sectional area of the headgroups and hence an increased critical packing parameter (CPP) of the complexes.

(ii) Figure 6 shows typical SAXS patterns obtained for the (a) C10Glu-C10DMA, (b) C12Glu-C12DMA, and (c) C14Glu-C14DMA systems at various temperatures at a given concentration. For example, we observed the $\left(\mathrm{H}_{1}+\right.$ $\left.\mathrm{V}_{1}\right)-\mathrm{V}_{1}-\mathrm{L}_{\alpha}$ phase transition with increasing temperature in the C10Glu-C10DMA system at a fixed concentration of 55 wt $\%$. These phase transitions reflect the decreased hydration around the headgroups and the amide bond, leading to the decreased cross-sectional area of the headgroups. Hence, the positive curvature becomes lower at high temperatures. We confirmed the similar behavior in the $\mathrm{Cn}$ Glu single system ${ }^{18)}$, the amino acid-based gemini surfactant system $^{18)}$, and the oleic acid-based gemini surfactant system $^{19)}$.

(iii) For understanding the effects of hydrophobic chain length, the phase diagrams in Fig. 4 were compared. First, the single $V_{1}$ phase is present in the C10Glu-C10DMA system, whereas this phase appears as mixture with other phases in the C12Glu-C12DMA and C14Glu-C14DMA systems. Second, the phase transitions occur at lower con- centrations for longer hydrophobic chain analogs. This behavior is predicted by the CPP; the shorter hydrophobic chain analog tends to form assemblies with greater positive curvature, whereas the longer hydrophobic chain analog tends to form assemblies with lesser positive curvature. Third, the $d$-spacing values of the $\mathrm{H}_{1}$ phase are shown in Table 2. The values become larger with increasing chain length. Fourth, the increased chain length also resulted in the narrower $\mathrm{L}_{\alpha}$ region and the wider solid-present region. This results from the increased hydrophobicity and hence the decreased solubility in water for the longer chain analogs.

(iv) Finally, the phase diagrams of the C12Glu-C12DMA and $\mathrm{C} 12 \mathrm{Glu}$ systems ${ }^{18)}$ were compared. In the case of the C12Glu-C12DMA system, the $\mathrm{L}_{1}-\mathrm{H}_{1}$ phase transition occurs and the $\mathrm{H}_{1}$ region appears in a wide range, whereas in the case of the C12Glu system, the discontinuous cubic liquid $\operatorname{crystal}\left(\mathrm{I}_{1}\right)$ phase appeared and $\mathrm{L}_{1}-\mathrm{I}_{1}$ phase transition occurred. Here, C12Glu-C12DMA consists of double-chains and a single-head, whereas C12Glu consists of a singlechain and double-heads. Clearly, the CPP of C12Glu-C12D$\mathrm{MA}$ is expected to be larger than that of $\mathrm{C} 12 \mathrm{Glu}$, and hence the assemblies having lesser positive curvature (such as $\mathrm{H}_{1}$ phase) are preferentially formed for C12Glu-C12DMA. 


\section{R. Yoshikawa, M. Akamatsu, K. Sakai et al.}

Table $2 d$-spacing values of the $\mathrm{H}_{1}$ phase at a fixed concentration of $40 \mathrm{wt} \%$.

\begin{tabular}{cc}
\hline Complex & $d(\mathrm{~nm})$ \\
\hline C10Glu-C10DMA & 4.08 \\
C12Glu-C12DMA & 4.80 \\
C14Glu-C14DMA & 5.57 \\
\hline
\end{tabular}

\section{Conclusions}

In this study, we characterized the physicochemical properties of $\mathrm{C} n \mathrm{Glu}-\mathrm{C} n \mathrm{DMA}$ in water at low and high concentrations. The static surface tensiometry revealed that the increased chain length of $\mathrm{C} n \mathrm{Glu}-\mathrm{C} n \mathrm{DMA}$ resulted in the decreased cmc, as generally observed in surfactant systems. The alkylamine ( $n$ DMA) plays a role as a counterion of $\mathrm{C} n \mathrm{Glu}$ as well as a hydrophobicity enhancer. This leads to the excellent surface activity, being comparable to gemini surfactant. In the aspect of the phase behavior at their high concentrations, we observed the $\mathrm{L}_{1}-\mathrm{H}_{1}-\mathrm{V}_{1}-\mathrm{L}_{\alpha}$ phase transition with increasing concentration. The increased temperature also yielded the assemblies with lesser positive curvature. The complex formation of $\mathrm{C} n \mathrm{Glu}$ with CnDMA induced the formation of assemblies with lesser positive curvature, and hence $\mathrm{C} n \mathrm{DMA}$ also acts as a curvature modifier of $\mathrm{C} n \mathrm{Glu}$.

\section{References}

1) Zana, R.; Xia, J. Gemini Surfactants, Synthesis, Interfacial and Solution-Phase Behavior, and Applications (Zana, R.; Xia, J. ed.). Marcel Dekker, New York. Chapter 1(2003).

2) Zana, R.; Alami, E. Novel Surfactants: Preparation, Applications, and Biodegradability (Holmberg, K. ed.). Marcel Dekker, New York. Chapter 12 (2003).

3) Sakai, H.; Okabe, Y.; Tsuchiya, K.; Sakai, K.; Abe, M. Catanionic mixtures forming gemini-like amphiphiles. J. Oleo Sci. 60, 549-555(2011).

4) Liu, D.; Suo, Y.; Tan, J.; Lu, H. $\mathrm{CO}_{2}$-Switchable microemulsion based on a pseudogemini surfactant. Soft Matter 13, 3783-3788(2017).

5) Wang, Z.; Ren, G.; Yang, J.; Xu, Z.; Sun, D. $\mathrm{CO}_{2}-\mathrm{Re}-$ sponsive aqueous forms stabilized by pseudogemini surfactants. J. Colloid Interface Sci. 536, 381-388 (2019).

6) Zhang, Y.; Feng, Y.; Wang, Y.; Li, X. $\mathrm{CO}_{2}$-Switchable viscoelastic fluids based on a pseudogemini surfactant. Langmuir 29, 4187-4192 (2013).

7) Noori, S.; Naqvi, A.Z.; Ansari, W.H.; Akram, M.; KabirUd-Dim, K. Synthesis and investigation of surface active properties of counterion coupled gemini surfac- tants. J. Surfactants Deterg. 17, 409-417 (2014).

8) Asadov, Z.H.; Huseynova, S.M.; Ahmadova, G.A.; Rahimov, R.A.; Sharbatov, S.U.; Zubkov, F.I.; Jafarova, R.A. Synthesis, colloidal-chemical and petroleum collecting properties of new counterion coupled gemini surfactants based on hexadecylbis (2-hydroxypropyl)amine and dicarboxylic acids. J. Dispers. Sci. Technol. e1-e9 (2019).

9) Chu, Z.; Feng, Y. pH-Switchable wormlike micelles. Chem. Commun. 46, 9028-9030 (2010).

10) Akong, F.O.; Pasc, A.; Emo, M.; Gérardin-Charbonnier, C. A supramolecular hydrogel based on an original pseudopeptidic catanionic surfactant. New J. Chem. 37, 559-562 (2013).

11) Hao, L.; Yegin, C.; Chen, I.C.; Oh, J.K.; Liu, S.; Scholar, E.; Zhang, L.; Akbulut, M.; Jiang, B. pH-Responsive emulsions with supramolecularly assembled shells. Ind. Eng. Chem. Res. 57, 9231-9239(2018).

12) Sakai, K.; Sawa, M.; Nomura, K.; Endo, T.; Tsuchiya, K.; Sakamoto, K.; Abe, M.; Sakai, H. pH-Sensitive wormlike micelle and hydrogel formation by acylglutamic acid-alkylamine complex. Chem. Lett. 45, 655-657 (2016).

13) Tojinbara, T.; Akamatsu, M.; Sakai, K.; Sakai, H. Oil-inwater emulsions stabilized by acylglutamic acid-alkylamine complexes as noncovalent-type double-chain amphiphiles. Langmuir 34, 268-272 (2017).

14） Sakai, K.; Nomura, K.; Shrestha, R.G.; Endo, T.; Sakamoto, K.; Sakai, H.; Abe, M. Wormlike micelle formation by acylglutamic acid with alkylamines. Langmuir 28, 17617-17622 (2012).

15) Takehara, M.; Moriyuki, H.; Yoshimura, I.; Yoshida, R. Surface active $N$-acylglutamate: II. Physicochemical properties of long chain $N$-Acylglutamic acids and their sodium salts. J. Am. Oil Chem. Soc. 49, 143-150 (1972).

16) Moulik, S.P.; Haque, M.E.; Jana, P.K.; Das, A.R. Micellar properties of cationic surfactants in pure and mixed states. J. Phys. Chem. 100, 701-708(1996).

17) Klevens, B.H. Critical micelle concentrations as determined by refraction. J. Phys. Colloid Chem. 52, 130148(1948).

18) Shrestha, R.G.; Nomura, K.; Yamamoto, M.; Yamawaki, Y.; Tamura, Y.; Sakai, K.; Sakamoto, K.; Sakai, H.; Abe, M. Peptide-based gemini amphiphiles: Phase behavior and rheology of wormlike micelles. Langmuir 28, 15472-15481 (2012).

19) Sakai, K.; Umemoto, N.; Aburai, K.; Takamatsu, Y.; Endo, T.; Kitiyanan, B.; Matsumoto, M.; Sakai, H.; Abe, M. Physicochemical properties of oleic acid-based partially fluorinated gemini surfactants. J. Oleo Sci. 63, 257-267(2014). 